\title{
Thoraco-abdominal aneurysm repair in Marfan patients
}

\author{
Pier Paolo Zanetti \\ Surgical Department, Center of Thoracic Aorta Surgery and Marfan Disease, Policlinico di Monza, \\ Monza (Milan), Italy
}

Kardiochirurgia i Torakochirurgia Polska 2012; 3: 292-294

Marfan syndrome is an autosomal dominant hereditary disease, which has an incidence of about 1 in 3000-5000 births.

This syndrome has been described by Antonio Bernardo Marfan, pediatrician in Paris Hospital, who visited a young woman with very long and tapering fingers (arachnodactyly) and massive articular weakness, and it remained undiscovered for many years.

This syndrome mainly presents in the $2^{\text {nd }}-4^{\text {th }}$ decade (young adults), more often in men according to some statistics, with equal incidence according to others.

Marfan disease is substantially due to a genetic mutation in autosomal chromosome 15 that causes incorrect production of a protein named fibrillin, which is one of the most important components of the extracellular matrix that builds elastin and elastic fibers.

In particular, this abnormality causes the inability to fix calcium, creating very weak connective tissue; eyes, osteoskeletal and cardiovascular apparatus are hardly involved, because they are very rich in connective tissue.

There are more than 100 possible mutations in chromosome 15 but one of these mutations is present in just $75 \%$ of real Marfan patients.

We can use GHENT criteria to define Marfan disease; these criteria are not about the presence of any mutation; they concern how many apparatuses (primary or secondary) are involved at the same time, with or without a familiar risk factor.

This syndrome is very variegated; in fact, we often encounter patients who do not present any physical sign of Marfan syndrome (Marfan stigmata) but have a typical Marfan cardiovascular apparatus; these patients are named "Marfan Frusti" and they deserve the same treatment as real Marfan patients.

Finally, $25 \%$ of Marfan patients have no familiar history of disease.

Now we know that Marfan syndrome mainly concerns eyes, osteo-skeletal and cardiovascular apparatus but just the last one is the most frequently involved as the principal cause of mortality and morbidity; in $75 \%$ of cases, the disease starts with aortic bulb and ascending aorta expansion in young adults, which are exposed to dissection and rupture. The remaining $25 \%$ of patients present aortic arch (10\%), descending aorta (9\%), thoraco-abdominal tract (5\%) and subrenal aortic tract (1\%) expansion.

In particular, we discovered that more than $90 \%$ of TAAs (thoraco-abdominal aneurysms) in Marfan patients present in subjects who have been previously treated for type A or type B dissection; we hypothesize that approximately $30-40 \%$ of patients who underwent ascending aorta or aortic arch replacement will have thoraco-abdominal aorta evolution in an aneurysmatic way. These patients will need many subsequent surgical procedures to repair the whole aortic wall; for this reason, it is so important to organize a very close follow-up of these patients, because we know (from Bonser publications) that aortic expansion will increase by about $0.36-0.40 \mathrm{~mm}$ per year if the starting diameter is $3.8-4 \mathrm{~cm}$, by about $0.60 \mathrm{~mm}$ per year if the diameter is $5 \mathrm{~cm}$, and by about $1.5 \mathrm{~cm} /$ year if the starting measure is $6 \mathrm{~cm}$.

As observed by Coselli, TAA in Marfan patients can present as an emergency, if a surgical procedure has to be performed immediately because of a clear rupture; they can also be an urgency, that can be treated in the time of hospital stay, if the patient is symptomatic or there is a covered rupture, or they can be elective, without any immediate risk of death; this category can undergo other diagnostic procedures.

Dissection can be defined as acute within 15 days from the beginning, chronic beyond 15 days. TAAs have been classified by Crawford and, as seen before, $80 \%$ of them are classified as aneurysm over dissection.

In our personal experience, we have operated on 613 TAA cases (from 1994 to 2011) and we found 39 cases that resulted from Marfan syndrome or "Marfan Frusti"; we observed that, even if we considered a very long period of

Address for correspondence: Prof. Pier Paolo Zanetti, Surgical Department, Center of Thoracic Aorta Surgery and Marfan Disease, Policlinico di Monza, Piazza Cinque Giornate 10, 20129 Milano, Italy, Email: pp.zanetti@policlinicodimonza.it 
time, surgical indication for these Marfan patients has not changed over time.

In any asymptomatic real Marfan patient, we operate on TAAs starting from $4.5 \mathrm{~cm}$ in diameter, if the expansion increases by $1-1.5 \mathrm{~cm}$ per year; in fact, in a real Marfan patient, surgical suggestion, even if it can appear prophylactic, seems to be totally applicable because of the young age of the patient, the evolutionary trend of this disease and the massive mortality and morbidity of the patients who undergo emergency procedures. This suggestion is even more justified if a familiar risk factor is present.

However, TAAs with a diameter starting from $6 \mathrm{~cm}$ or more are an absolute indication for a surgical procedure, regardless of annual increase. Even symptomatic patients or covered and clear ruptures represent situations that obviously deserve an immediate surgical approach.

It is very important to emphasize that there is $30-40 \%$ morbidity and mortality in patients who undergo an emergency or urgent surgical procedure; with this in mind, we suggest treating TAA as soon as possible, even because this indication is largely shared, as confirmed by several scientific publications [1-4].

From our experience in treating Marfan patients, we suggest extreme delicacy and attention in preparing and performing surgical procedures, because these subjects often show some other important thoracic malformations or have undergone previous surgical procedures.

$70 \%$ of TAAs that we treated were type I or II, according to Crawford's classification, and during procedures we used Bio-Pump or extracorporeal circulation (ECC), liquor drainage and aggressive reimplantation of intercostal arteries. Regarding Bio-Pump or ECC, only in 3 of our 39 Marfan cases (9\%) did we need ECC with deep hypothermia and cardiac arrest (DHCA); in two of the cases this procedure was necessary because we could not clamp proximally, due to the distal aortic arch that was involved in expansion. In the third case, there was an endoprosthesis in loco that prevented proximal clamping.

We use HPCA only in extreme cases, because it requires complete heparinization, which complicates good hemostasis, especially when there is left T.L.F., and can cause post-surgical bleeding. Additionally, we saw many breathing and coagulative troubles after this procedure. From our experience, even if three cases are maybe too few, we observed no mortality nor morbidity related to HPCA, maybe due to the young age of the patients.

Bio-Pump is our preferred method for the treatment of type I, II, III TAA (according to Crawford classification); we choose "Clamp and go" technique for type IV TAA.

Talking about cannulae application, from case to case we use the left inferior pulmonary vein, left superior pulmonary vein or left atrium; on the arterial side, we use the common femoral artery or aneurysmatic aorta. Proximal clamping is applied between the left common carotid artery and left subclavian artery, but in four cases we clamped between the anonymous artery and left common carotid artery, after placing the patient in a Trendelenburg position and maintaining arterial pressure higher than 130-140 mm $\mathrm{Hg}$. This procedure requires speed in performing the proximal suture line, correct anatomy and functioning of Willis circle and young age of the patient.

Liquor drainage has to be considered a routine procedure, not applicable just in case of hemodynamic instability for clear rupture, septic status, decoagulated patients or previous surgical procedures on the spinal column. Drainage is placed in the operating room and removed two days after, monitoring the pressure to keep values between 10 and $15 \mathrm{mmHg}$.

In Marfan patients, intercostal arteries, that result open, are numerous because of the young age of the subjects and they often emerge not only from the true lumen but also from the false one. In this case, we always decide to remove the septum between the two lumina and reimplant long segments of the posterior aortic wall (where intercostal arteries emerge); after this procedure, there is a high risk of pseudoaneurysm in the long term. Finally, we normally close the intercostal arteries between T3 and T9, not directly implicated in developing paraparesis/paraplegia. Sometimes, it is very complicated to control run-off hemorrhages, which can be severe.

Concerning other technical notes, we need to perform the suture line strengthened with pledgets, especially for renal and visceral vessels, because of aortic wall tenderness. Then, we also use Ringer lactate perfusion and a special prosthesis (developed by Coselli), which presents four branches that fit together with ostia of the vessels; in this way, we can cut off the weak aortic wall from stitches, lowering the possibility of pseudoaneurysm in the long term.

Looking at the rate of mortality and morbidity in Marfan patients who present TAA, if we compare them to nonMarfan patients, we can observe that these data are better in Marfan patients, both in elective and emergency procedures. Obviously, rates are better in elective procedures; but if we look at the emergency procedures, percentages of mortality and morbidity are very close between Marfan and non-Marfan patients. This result may be due to the younger age of Marfan patients; and also because non-Marfan patients undergo surgery during the $5-7^{\text {th }}$ decade and they are often affected by some other comorbidities such as diabetes mellitus, arterial hypertension, coronaropathies, supra-aortic vessel atheromata and COPD.

We have to specify that Marfan patients often need to undergo many surgical procedures, because of the progressive trend of this disease, which often requires the removal of the whole aortic wall.

Endoprosthesis (thoracic endovascular aortic repair TEVAR) in Marfan patients affected by TAA is controversial, both in our experience and according to international scientific publications.

Use of TEVAR in Marfan can be dangerous because of extreme frailty of the aortic wall and the progressive trend of this disease. One of the most problematic procedures in using TEVAR is the proximal fixing of the prosthesis, because we can cause aortic wall rupture or immediate and 
long-term dissection, also type non-A non-B. Even expansion with a balloon can be very dangerous, because of possible aortic wall damage.

Other possible complications related to TEVAR are expansions of the aortic wall in the landing zone, because we can produce proximal and distal leaks, with subsequent reperfusion and evolution of the aneurysmatic dilatation, that can result in unexpected ruptures; besides, if there is a total aortic wall expansion in the landing zone, we can have detachment and migration of our device, which can cause dramatic ischemic events, especially in the spinal cord, visceral, renal and inferior limb vessels.

In our experience, upstream and downstream of the prosthesis we frequently discover aneurysmatic expansions, caused by aortic wall weakness; we also observed extreme difficulty in surgical removal of a migrated or suppurated prosthesis, with subsequent complicated aortic reconstructions and high rates of mortality and morbidity.

Beyond these observations, in Marfan patients there are some situations that need endoprosthesis TEVAR: subjects with previous multiple surgical procedures on the chest or on the sternum, massive bleeding risk, possibility of lung and/or heart damage, and high risk of mortality.

One other possibility is the "focal" lesion as the pseudoaneurysms on the suture lines or proximal and distal small expansion after aortic wall replacement. As reported by Coselli, surgical procedures for endoprosthesis removal are very demanding and complicated, with great difficulty in aortic wall reconstruction and high percentages of mortality and morbidity.

In conclusion, we mostly recommend, for TAA treatment in Marfan patients, "open surgery" versus endoprosthesis with TEVAR. From our experience, in these subjects we suggest a very close follow-up of thoraco-abdominal aortic diameters because, since these patients are often very young with a progressive disease, they could require surgery very soon, even in a prophylactic way, because mortality and morbidity are almost prohibitive in patients who undergo urgent or emergency surgical treatment

During the operations on Marfan patients, we have to replace the diseased aortic wall in total: surgery has to be as extensive as it can be; in fact, incomplete aortic wall replacements can lead to subsequent multiple surgical procedures because of possible involvement of a remaining segment of untreated aorta. Also, percentages of mortality and morbidity can increase, because of prosthesis infections, especially in patients with immune system depression.

The surgical follow-up of our patients comprises computed tomography or magnetic nuclear resonance every 3-6-12 months; survival at one year is about $90 \%$, and at five years is about $75 \%$.

These data are very flattering, especially because, before the 1970s and before the cardiovascular approach to Marfan disease, survival of these patients was about 30-40 years; now, they can quite easily reach 70 years - the same age as the non-affected population.

\section{References}

1. Milewicz DM, Dietz HC, Miller DC. Treatment of aortic disease in patients with Marfan syndrome. Circulation 2005; 111: e150-157.

2. LeMaire SA, Carter SA, Volguina IV, Laux AT, Milewicz DM, Borsato GW, Cheung CK, Bozinovski J, Markesino JM, Vaughn WK, Coselli JS. Spectrum of aortic operations in 300 patients with confirmed or suspected Marfan syndrome. Ann Thorac Surg 2006; 81: 2063-2078;

3. Kalkat MS, Rahman I, Kotidis K, Davies B, Bonser RS. Presentation and outcome of Marfan's syndrome patients with dissection and thoraco-abdominal aortic aneurysm. Eur J Cardiothorac Surg 2007; 32: 250-254.

4. Mommertz G, Sigala F, Langer S, Koeppel TA, Mess WH, Schurink GW, Jacobs MJ. Thoracoabdominal aortic aneurysm repair in patients with marfan syndrome. Eur J Vasc Endovasc Surg 2008; 35: 181-186. 